\title{
Interactions between leptin and insulin resistance in patients with prediabetes, with and without NAFLD
}

\author{
SIMONA BUNGAU ${ }^{1}$, TAPAN BEHL $^{2 *}$, DELIA MIRELA TIT ${ }^{1}$, FLORIN BANICA $^{1 *}$, \\ OVIDIU G. BRATU ${ }^{3}$, CAMELIA C. DIACONU ${ }^{4}$, CARMEN D. NISTOR-CSEPPENTO ${ }^{5}$, \\ CRISTIANA BUSTEA ${ }^{6 *}$, RALUCAA. CORB ARON ${ }^{6}$ and COSMIN MIHAI VESA ${ }^{6}$
}

\begin{abstract}
${ }^{1}$ Department of Pharmacy, Faculty of Medicine and Pharmacy, University of Oradea, 410073 Oradea Romania; ${ }^{2}$ Chitkara College of Pharmacy, Chitkara University, 140401 Punjab, India; ${ }^{3}$ Clinical Department 3 and ${ }^{4}$ Department 5,

'Carol Davila' University of Medicine and Pharmacy, 050474 Bucharest; Departments of ${ }^{5}$ Psycho-Neurosciences and Recovery, and ${ }^{6}$ Preclinical Disciplines, Faculty of Medicine and Pharmacy, University of Oradea, 410073 Oradea, Romania
\end{abstract}

Received July 10, 2020; Accepted August 12, 2020

DOI: $10.3892 /$ etm.2020.9327

\begin{abstract}
This study explored the link between insulin sensitivity, insulin resistance and leptinaemia in people with prediabetes with and without non-alcoholic fatty liver disease (NAFLD). A total of 143 prediabetes patients were evaluated in the study. Ultrasonography was used for diagnosis of NAFLD, and fasting insulin, postprandial insulin, leptin levels, common clinical/biochemical determinations were determined. In total, $69(48.25 \%)$ of the patients were diagnosed with NAFLD and $74(51.75 \%)$ without NAFLD. Leptin values correlated statistically with fasting insulin in prediabetes patients, while in the patients that were also diagnosed with NAFLD the correlation was stronger. Values of log-leptin $<1 \mathrm{ng} / \mathrm{ml}$ were found in $64 \%$ of patients with prediabetes without NAFLD, and in $2 \%$ of patients with prediabetes and NAFLD. In the context of the association between serum leptin levels and a worse biochemical profile in prediabetes patients on one hand, and fatty liver disease and a worse biochemical profile in prediabetes patients on the other, leptin can be considered a possible candidate molecule that mediates the biochemical alterations identified in these patients.
\end{abstract}

\section{Introduction}

Non-alcoholic fatty liver disease (NAFLD) represents an accumulation of fatty acids in the liver usually diagnosed in patents with obesity because of altered insulin singling and

Correspondence to: Dr Ovidiu G. Bratu, Clinical Department 3, 'Carol Davila' University of Medicine and Pharmacy, 8 Eroii Sanitari Blvd., 050474 Bucharest, Romania

E-mail: ovi78doc@yahoo.com

${ }^{*}$ Contributed equally

Key words: insulin, leptin, non-alcoholic fatty liver, prediabetes, interactions releasing of free fatty acids from the adipose tissue (1). Obesity is the factor that determines the initiation of insulin-resistance, a precursor of prediabetes and also type 2 diabetes mellitus (T2DM) (2). Because NAFLD and prediabetes share the same substrate, insulin-resistance, their association is frequently encountered in clinical practice $(3,4)$.

Leptin is a cytokine produced by the adipocytes. Its main role is to regulate the energy balance and body weight by acting on the hypothalamus (5). Although it should reduce appetite in obese and overweight patients (susceptible to prediabetes and T2DM development), the serum levels of leptin are higher than in normal-weight individuals (6). Hyperleptinemia is considered a consequence of leptin resistance in the central or peripheral nervous system. Leptin interactions are relevant in the glucose metabolism alterations, considering the important relationships of leptin and insulin $(5,7)$.

The purpose of this study was to analyze the interactions between leptin and insulin resistance in prediabetes patients, in the presence or absence of NAFLD. The association of NAFLD, leptin, prediabetes has rarely been explored in literature.

\section{Patients and methods}

The study was performed in the Clinical County Emergency Hospital of Oradea (Oradea, Romania). A total of 143 prediabetes patients, aged 18-75 years were evaluated in the study, between February 1, 2018-February 1, 2020. The criteria for prediabetes diagnosis was a HbA1c level of 5.7 to $6.4 \%$ (according to the guideline of American Diabetes Association (7) that established the reference values for prediabetes diagnosis). Patients' exclusion criteria were as follows: with DM, under treatment with steroid drugs (known to increase glycaemia), suffering from alcoholism, with liver cirrhosis, etc. In total, 614 subjects were evaluated in the above specified circumstances for DM suspicion (263 being diagnosed); 187 patients without diabetes/prediabetes were excluded based on $\mathrm{HbA1c}<5.7 \%$; 164 patients remained potential candidates for this study (HbAlc between 5.7-6.4\%). 21 more patients were excluded after applying the above exclusion criteria. Thus, the 143 individuals who remained 
Table I. Frequency of log-leptin value distribution in non-NAFLD/NAFLD patients. ${ }^{\text {a }}$

\begin{tabular}{|c|c|c|c|c|c|c|c|c|c|}
\hline \multicolumn{2}{|c|}{ Log-leptin (ng/ml) } & \multicolumn{2}{|c|}{ Count } & \multicolumn{2}{|c|}{ Cumulative count } & \multicolumn{2}{|c|}{$\%$} & \multicolumn{2}{|c|}{ Cumulative $\%$} \\
\hline nNFDL & NFDL & nNFDL & NFDL & nNFDL & NFDL & nNFDL & NFDL & nNFDL & NFDL \\
\hline $0.2-0.4$ & \multirow{4}{*}{$\begin{array}{r}0.6-0.8 \\
1-1.2\end{array}$} & 9.00 & \multirow[b]{3}{*}{4.00} & 9.00 & \multirow[b]{3}{*}{4.00} & 0.12 & \multirow{4}{*}{$\begin{array}{l}0.06 \\
0.28\end{array}$} & 0.12 & \multirow[b]{3}{*}{0.06} \\
\hline $0.4-0.6$ & & 11.00 & & 20.00 & & 0.15 & & 0.27 & \\
\hline $0.6-0.8$ & & 15.00 & & 35.00 & & 0.20 & & 0.47 & \\
\hline $0.8-1$ & & 8.00 & 19.00 & 43.00 & 23.00 & 0.11 & & 0.58 & 0.34 \\
\hline $1-1.2$ & \multirow{3}{*}{$\begin{array}{l}1.2-1.4 \\
1.4-1.6 \\
1.6-1.8\end{array}$} & 7.00 & \multirow{3}{*}{$\begin{array}{r}26.00 \\
14.00 \\
6.00\end{array}$} & 50.00 & \multirow{3}{*}{$\begin{array}{l}49.00 \\
63.00 \\
69.00\end{array}$} & 0.10 & \multirow{3}{*}{$\begin{array}{l}0.38 \\
0.20 \\
0.08\end{array}$} & 0.68 & 0.72 \\
\hline $1.2-1.4$ & & 9.00 & & 59.00 & & 0.12 & & 0.80 & 0.92 \\
\hline $1.4-1.6$ & & 9.00 & & 68.00 & & 0.12 & & 0.92 & 1.00 \\
\hline $1.6-1.8$ & & 6.00 & & 74.00 & & 0.08 & & 0.06 & \\
\hline
\end{tabular}

${ }^{a} \mathrm{nNFDL}$, non NAFLD patients $(\mathrm{n}=74)$; NAFLD patients ( $\left.\mathrm{n}=69\right)$. NAFLD, non-alcoholic fatty liver disease.

in the study were evaluated by performing clinical exam and the basic biochemical determination. Additionally, serum insulin and leptin were determined in a private laboratory (SC Biostandard 2007 Srl, Oradea, Romania). NAFLD diagnosis was based on the presence of increased liver echogenicity, in the absence of alcohol intake. The diagnosis of NAFLD was made in the radiology department of the hospital with the help of $3.5 \mathrm{MHz}$ linear transducer. Among the total patients with prediabetes, 74 patients $(51.75 \%)$ were without NAFLD, and 69 patients $(48.25 \%)$ with NAFLD, based on the ultrasonography. Insulin was determined by the immunochemical method with electrochemiluminescence detection (ECLIA); leptin was determined by ELISA method. HOMA-IR, HOMA\%B and $\mathrm{HOMA} \% \mathrm{~S}$ were determined using the values of fasting glucose (G0), fasting insulin (INS0), post-prandial glucose (G120) and post-prandial insulin (INS120). Leptin values were expressed as the logarithm 'log-leptin', since leptin is a variable with highly skewed distribution.

Our study was conducted according to the WMA Declaration of Helsinki, Ethical Principles for Medical Research Involving Human Subjects; it was also approved by the Ethics Committee of the Clinical County Emergency Hospital of Oradea (Oradea, Romania). All the patients included in the study signed an informed consent.

SPSS v. 17 software (SPSS, Inc.) was used for statistical analysis. Anova test and the Mann-Whitney test were applied for the numerical variables, to obtain the $\mathrm{P}$-value $(\mathrm{P}<0.05$ was considered to indicate a statistically significant difference); moreover, Chi-square test for proportions was used for the qualitative variables.

\section{Results}

Compared with patients with prediabetes but without NAFLD, patients with prediabetes and NAFLD had values that were statistically significant to a higher degree for: systolic blood pressure (SBP) $(131.45 \pm 33.02$ vs. $112.74 \pm 14.50 \mathrm{mmHg}, \mathrm{P}<0.01)$, diastolic blood pressure (DBP) $(88.97 \pm 23.62$ vs. $75.74 \pm 17.58 \mathrm{mmHg}, \mathrm{P}<0.01)$, waist circumference $(93.30 \pm 7.23$ vs. $88.27 \pm 8.38 \mathrm{~cm}, \mathrm{P}<0.01)$, triglycerides (TG) $(209.73 \pm 137.18$ vs. $153.38 \pm 80.44 \mathrm{mg} / \mathrm{dl}$,
$\mathrm{P}<0.02)$, glutamic-pyruvic transaminase (GPT) $(38.80 \pm 18.43$ vs. $31.63 \pm 13.10 \mathrm{IU} / \mathrm{l}, \mathrm{P}=0.02), \gamma$-glutamyl transferase $(\mathrm{GGT})$ (33.57 \pm 12.76 vs. $26.85 \pm 13.44 \mathrm{IU} / 1, \mathrm{P}=0.01)$, glutamic oxaloacetic transaminase (GOT) $(35.69 \pm 22$ vs. $26 \pm 8.91)$, insulin resistance $(4.49 \pm 1.93$ vs. $3.52 \pm 0.93, \mathrm{P}<0.01)$, log-transformed leptin $(1.28 \pm 0.18$ vs. $0.86 \pm 0.40, \mathrm{P}<0.01)$, and values that were statistically significant to a lower degree for HDL-C (34.35 \pm 7.61 vs. $38.49 \pm 7.58 \mathrm{mg} / \mathrm{dl}, \mathrm{P}<0.01), \beta$-cell function $(109.84 \pm 34.04$ vs. $130.14 \pm 34.86, \mathrm{P}<0.01)$ and insulin sensitivity $(45.65 \pm 14.20$ vs. $58.90 \pm 21.55, \mathrm{P}<0.01)$. There was no statistically significant difference concerning prediabetes categories in the groups with or without NAFLD. Impaired glucose tolerance was identified in $21.42 \%$ of patients with NAFLD and in $20 \%$ of patients without NAFLD; impaired fasting glucose was identified in $40.47 \%$ of patients with NAFLD and in $47.27 \%$ of patients without NAFLD; the association between impaired glucose tolerance and impaired fasting glucose was found in $38.09 \%$ of patients with NAFLD and in $32.2 \%$ of patients without NAFLD.

A possible association was also tested between the leptin values and the fasting insulin $(\mu \mathrm{IU} / \mathrm{ml})$ values, respectively, between the leptin and the postprandial insulin $(\mu \mathrm{IU} / \mathrm{ml})$ values for the entire sample, respectively, only for the patients with NAFLD. A linear regression model was applied, considering the leptin values as predictors; a positive association resulted in all cases, being significant only in the case of leptin and fasting insulin $(r=0.35,=0.12, p=0.0004<0.05)$ association. Interestingly, when the same hypothesis was tested on the NAFLD patients, a better association between leptin and fasting insulin $(r=0.41,=0.17, p=0.006)$ was obtained. So, it can be stated that the leptin values can be linked to the fasting insulin values. Table I shows that a greater proportion of patients with NAFLD are distributed in the higher intervals of log-leptin values than in the patients without NAFLD. A value of log-leptin $<1 \mathrm{ng} / \mathrm{ml}$ was found in $64 \%$ of patients with prediabetes without NAFLD, and in $2 \%$ of patients with prediabetes and NAFLD. Also, risk analysis was performed to see if the presence of NAFLD increases the chances of deterioration of medical tests/results as blood pressure or HOMA-IR, HOMA\%B and HOMA\%S. If this link would be proven, it would indicate whether a patient with NAFLD has a 
Table II. Risk analysis.

\begin{tabular}{|c|c|c|c|c|}
\hline \multirow{2}{*}{$\frac{\text { Exposure+/- }}{\text { Scenarios }}$} & \multirow{2}{*}{$\frac{\text { Disease presence }}{\mathrm{SBP} 130 \mathrm{~mm} \mathrm{Hg}}$} & \multicolumn{3}{|l|}{ Disease absence } \\
\hline & & $\mathrm{SBP} \leq 130 \mathrm{~mm} \mathrm{Hg}$ & Patients & Statistics \\
\hline \multicolumn{5}{|l|}{ Scenario 1} \\
\hline NAFLD+ & 21 & 48 & 69 & $\mathrm{P}=0.000025$ \\
\hline NAFLD- & 3 & 71 & 74 & $\mathrm{OR}=10.35$ \\
\hline Total & 24 & 119 & 143 & $\mathrm{OR} \in(2.93 ; 36.65)$ \\
\hline Scenario 2 & $\mathrm{DBP}>80 \mathrm{~mm} \mathrm{Hg}$ & $\mathrm{DBP} \leq 80 \mathrm{~mm} \mathrm{Hg}$ & - & - \\
\hline NAFLD+ & 23 & 46 & 69 & $\mathrm{P}=0.00028$ \\
\hline NAFLD- & 6 & 68 & 74 & $\mathrm{OR}=5.66$ \\
\hline Total & 29 & 114 & 143 & $\mathrm{OR} \in(2.14 ; 14.99)$ \\
\hline Scenario 3 & HOMA-IR >4 & HOMA-IR $\leq 4$ & - & - \\
\hline NAFLD+ & 31 & 38 & 69 & $P=0.00009$ \\
\hline NAFLD- & 11 & 63 & 74 & $\mathrm{OR}=4.67$ \\
\hline Total & 42 & 101 & 143 & ORE $(2.10 ; 10.36)$ \\
\hline Scenario 4 & НОМА $\%$ В $<122$ & НОМА $\%$ В $\geq 122$ & - & - \\
\hline NAFLD+ & 44 & 25 & 69 & $\mathrm{P}=0.0043$ \\
\hline NAFLD- & 29 & 45 & 74 & $\mathrm{OR}=2.73$ \\
\hline Total & 73 & 70 & 143 & ORE $(1.39 ; 5.38)$ \\
\hline Scenario 5 & HOMA $\%$ S >53 & HOMA $\% \mathrm{~S} \leq 53$ & - & - \\
\hline NAFLD+ & 40 & 29 & 69 & $\mathrm{P} \leq 0.001$ \\
\hline NAFLD- & 12 & 62 & 74 & $\mathrm{OR}=7.13$ \\
\hline Total & 52 & 91 & 143 & $\mathrm{OR} \in(3.26 ; 15.59)$ \\
\hline
\end{tabular}

NAFLD, non-alcoholic fatty liver disease; DBP, diastolic blood pressure; OR, odds ratio.

higher chance of developing the disease than the other prediabetic patients. After applying the risk analysis and calculating the odds ratio parameter, a major statistically significant risk factor was obtained in all 5 tested scenarios (OR $>1,95 \%$ $\mathrm{OR}>1, \mathrm{P}<0.05)$. The $\mathrm{P}$-values from the chi square test were calculated in the EPI Info program using the Fischer exact formula. For the HOMA-IR, HOMA\%B and the HOMA\%S values we considered the integer mean value of our sample as cut off point. The detailed data are presented in Table II.

\section{Discussion}

Leptin is a relevant adipokine involved in the regulation of food intake (8), manifesting physiological action of inhibiting food intake by acting on certain brain receptors (9). Although its levels in obese individuals should reduce their appetite, most studies report that in reality leptin levels are higher in these individuals than in the lean individuals and do not manage reducing their food intake. In this investigation, a statistically significant correlation was found in univariate analysis between increased body-mass index and higher leptin levels, thereby confirming the associations reported in literature. Leptin could be the factor that promotes hepatic steatosis and fibrosis by up-regulation TNF- $\beta$ in the liver (10), acting as profibrotic cytokine; also, it could be the mediator (or at least one of the mediators) that explains the association between obesity and the occurrence of NAFLD. Another association found in patients with prediabetes was the statistically significant $(\mathrm{P}<0.01)$ correlation between serum leptin and insulin resistance, fact reported in previous studies (11). Leptin increases the sensitivity of cells to insulin (11), this being proof that in patients with prediabetes there is resistance to leptin action leading to increased insulin levels. The blood concentration of insulin and leptin in obese patients is high, being demonstrated experimentally that insulin stimulates the production and the secretion of leptin by the adipose cells (12). On the contrary, high insulin levels impair the physiological hypothalamic response to leptin for reducing appetite; weight loss helps to improve this response (13). Thus, overweight and obesity themselves generate weight accumulation leading to a vicious cycle.

In this study, high levels of leptin were associated with decreased insulin sensitivity in prediabetes patients. Under certain physiological conditions, leptin increases insulin sensitivity (14), thereby confirming the existence of an altered mechanism of action of this molecule in patients with prediabetes, obesity or overweight. Such associations have been reported in earlier studies, leading to high level of leptin to be considered a predictor of T2DM development (11), contrary to all the physiological roles that leptin fulfils in normal weight normoglycemic patients. There are many explanations why leptin resistance occurs, such as mutations of the leptin receptors, mutation of neurons in the hypothalamus that respond to leptin, and altered leptin signaling pathways 
but the most interesting mechanism is the altered transport of leptin through the blood-brain barrier (11). It seems that high levels of triglycerides inhibit this leptin transport (15) and that these high levels of triglycerides are specific to patients with NAFLD. Patients with NAFLD and prediabetes had triglyceride values that were higher to a statistically significant degree than prediabetes patients without NAFLD. There could be the following pathogenic links: Obesity promotes insulin resistance; high levels of insulin increase leptin levels; leptin cannot lead to decreased insulin levels and decreased appetite because of leptin resistance in the nervous system and in the adipose tissue; and high levels of leptin promote hepatic steatosis which in turn increases insulin resistance. Therefore, insulin and leptin play an important role in the development of prediabetes and NAFLD. Once NAFLD has appeared, high levels of triglycerides and increased hepatic insulin resistance aggravate the leptin abnormalities.

The risk analysis performed in this study confirmed that the presence of NAFLD implies major changes in the behavior of prediabetes patients in a negative way. By evaluating the differences between prediabetes patients with/without NAFLD, our results revealed that there are many significant alterations of the metabolic parameters. Thus, patients with prediabetes and NAFLD are exposed to a significantly higher cardiovascular risk and meet the criteria for metabolic syndrome. The patients that already have prediabetes and NAFLD present a significant risk of progression toward T2DM compared with patients without NAFLD, with $12.5 \%$ incidence of T2DM, after 10 years, in the presence of NAFLD and $2.5 \%$ incidence of T2DM in the absence of NAFLD (13). NAFLD increases cardiovascular risk and the risk of other comorbidities, chronic kidney disease (16) and dementia (which are chronic pro-inflammatory diseases as inferred by the increase in interlekin-6, fibrinogen, C-reactive protein, tissue plasminogen activators, and thus contribute to the atherogenesis process) (17). In the present study, one association of prediabetes patients is between NAFLD and the values of SBP and DBP, the same correlation being reported in previous studies (18). In patients with NAFLD, high concentrations of leptin have been observed together with diminished levels of adiponectin (19). Also, the degree of severity of NAFLD correlated with the levels of serum leptin (20). These consequences (dyslipidaemia, insulin resistance, chronic low-grade inflammation and NAFLD) have been investigated for favorable effects of a variety of natural phytochemicals or other antidiabetic drugs $(4,21)$. Finally, it has been demonstrated that leptin promotes the proliferation of hepatocellular carcinoma cells, a serious complication of NAFLD, while adiponectin administration suppresses this proliferation $(22,23)$.

The prevalence of NAFLD in patients with prediabetes was $48.25 \%$. The two conditions influence each other: NAFLD promotes insulin resistance and prediabetes, while prediabetes and insulin resistance promote lipid accumulation and fibrosis of the liver, the linking molecules between the two conditions being leptin. In the context of increased BMI, both insulin and leptin have high values, contrary to the expected normal mechanism of downregulation of insulin levels by circulating leptin. These results indicate a clear direction for further exploration with relevant possible confirmations whether leptin resistance is necessary in the development of T2DM, respectively NAFLD. Moreover, the existence of NAFLD in the prediabetes patients favors the development of other secondary diseases.

\section{Acknowledgements}

Not applicable.

\section{Funding}

No funding was received.

\section{Availability of data and materials}

Data are registered at the Clinical County Emergency Hospital of Oradea, Bihor County, Romania and SC Biostandard 2007 Srl (Oradea, Romania).

\section{Authors' contributions}

FB, OGB, CCD, CDNC, CB and RACA collected, analyzed and interpreted the patient data and the laboratory results. SB, TB, DMT and CMV made substantial contributions to the conception of the study and the interpretation of the data, drafted the manuscript and were major contributors in the writing of the manuscript. All authors read and approved the final manuscript to be published. All the authors agreed to be accountable for all aspects of the work in ensuring that questions related to the accuracy or integrity of any part of the work are appropriately investigated and resolved.

\section{Ethics approval and consent to participate}

The study was conducted according to the WMA Declaration of Helsinki, Ethical Principles for Medical Research Involving Human Subjects. The study was also approved by the Ethics Committee of the Clinical County Emergency Hospital of Oradea (Oradea, Romania). All the patients included in the study signed an informed consent.

\section{Patient consent for publication}

Not applicable.

\section{Competing interests}

The authors declare that they have no competing interests.

\section{References}

1. Caldwell S and Argo C: The natural history of non-alcoholic fatty liver disease. Dig Dis 28: 162-168, 2010.

2. Portillo-Sanchez P, Bril F, Maximos M, Lomonaco R, Bermacki D, Orsak B, Subbarayan S, Webb A, Hecht J and Cusi K: High prevalence of nonalcoholic fatty liver disease in patients with type 2 diabetes mellitus and normal plasma aminotransferase levels. J Clin Endocrinol Metab 100: 2231-2238, 2015.

3. Remus Popa A, Fratila O, Rus M, Corb Aron RA, Mihai Vesa C, Pantis C, Diaconu CC, Bratu O, Bungau S and Nemeth S: Risk factors for adiposity in the urban population and influence on the prevalence of overweight and obesity. Exp Ther Med 20: 129-133, 2020. 
4. Vesa CM, Popa L, Popa AR, Rus M, Zaha AA, Bungau S, Tit DM, Corb Aron RA and Zaha DC: Current data regarding the relationship between type 2 diabetes mellitus and cardiovascular risk factors. Diagnostics (Basel) 10: 314, 2020.

5. Zhou Y and Rui L: Leptin signaling and leptin resistance. Front Med 7: 207-222, 2013

6. Ernst MB, Wunderlich CM, Hess S, Paehler M, Mesaros A, Koralov SB, Kleinridders A, Husch A, Münzberg H, Hampel B, et al: Enhanced Stat 3 activation in POMC neurons provokes negative feedback inhibition of leptin and insulin signaling in obesity. J Neurosci 29: 11582-11593, 2009.

7. American Diabetes Association: 2. Classification and diagnosis of diabetes: Standards of medical care in diabetes-2020. Diabetes Care 43 (Suppl 1): S14-S31, 2020.

8. Margetic S, Gazzola C, Pegg GG and Hill RA: Leptin: A review of its peripheral actions and interactions. Int J Obes Relat Metab Disord 26: 1407-1433, 2002.

9. Myers MG Jr, Leibel RL, Seeley RJ and Schwartz MW: Obesity and leptin resistance: Distinguishing cause from effect. Trends Endocrinol Metab 21: 643-651, 2010.

10. Ikejima K, Okumura K, Lang T, Honda H, Abe W, Yamashina S, Enomoto N, Takei Y and Sato N: The role of leptin in progression of non-alcoholic fatty liver disease. Hepatol Res 33: 151-154, 2005.

11. Moonishaa TM, Nanda SK, Shamraj M, Sivaa R, Sivakumar P and Ravichandran K: Evaluation of leptin as a marker of insulin resistance in type 2 diabetes mellitus. Int J Appl Basic Med Res 7: 176-180, 2017.

12. Tiwari-Heckler S, Gan-Schreier H, Stremmel W, Chamulitrat W and Pathil A: Circulating phospholipid patterns in NAFLD patients associated with a combination of metabolic risk factors. Nutrients 10: 649, 2018.

13. Lustig RH, Sen S, Soberman JE and Velasquez-Mieyer PA: Obesity, leptin resistance, and the effects of insulin reduction. In J Obes Relat Metab Disord 28: 1344-1348, 2004.

14. Paz-Filho G, Mastronardi C, Wong ML and Licinio J: Leptin therapy, insulin sensitivity, and glucose homeostasis. Indian J Endocrinol Metab 16 (Suppl 3): S549-S555, 2012.
15. Popa AR, Bungau S, Vesa CM, Bondar AC, Pantis C, Maghiar O, Dimulescu IA, Nistor-Cseppento DC and Rus M: Evaluating the efficacy of the treatment with benfotiamine and alpha-lipoic acid in distal symmetric painful diabetic polyneuropathy. Rev Chim 70: 3108-3114, 2019.

16. Moisi IM, Rus M, Bungau S, Zaha CD, Uivarosan D, Fratila O, Tit DM, Endres L, Nistor-Cseppento DC and Popescu MI: Acute coronary syndromes in chronic kidney disease: Clinical and therapeutic characteristics. Medicina (Kaunas) 56: 118, 2020

17. Misra VL, Khashab M and Chalasani N: Nonalcoholic fatty liver disease and cardiovascular risk. Curr Gastroenterol Rep 11: $50-55,2009$.

18. Ryoo JH, Suh YJ, Shin HC, Cho YK, Choi JM and Park SK: Clinical association between non-alcoholic fatty liver disease and the development of hypertension. J Gastroenterol Hepatol 29: 1926-1931, 2014.

19. Adolph TE, Grander C, Grabherr F and Tilg H: Adipokines and non-alcoholic fatty liver disease, multiple interactions. Int J Mol Sci 18: 1649, 2017.

20. Lee Y, Kwon EY and Choi MS: Dietary isoliquiritigenin at a low dose ameliorates insulin resistance and NAFLD in diet-induced obesity in C57BL/6J mice. Int J Mol Sci 19: 3281, 2018.

21. Tacelli M, Celsa C, Magro B, Giannetti A, Pennisi G, Spatola F and Petta S: Drugs in NAFLD: The accomplishment of two goals at once? Pharmaceuticals (Basel) 11: 121, 2018.

22. Sharma D, Wang J, Fu PP, Sharma S, Nagalingam A, Mells J, Handy J, Page AJ, Cohen C, Anania FA and Saxena NK: Adiponectin antagonizes the oncogenic actions of leptin in hepatocellular carcinogenesis. Hepatology 52: 1713-22, 2010.

23. Diaconu C, Salmen T, Gaman MA, Bratu OG, Mischianu D, Marcu RD, Suceveanu AI, Costache RS and Pantea Stoian A: SGLT2 inhibition in patients with type 2 diabetes and cardiovascular diseases: Which are the benefits? Rom J Mil Med 122. 16-21, 2019. 